\title{
Sostenibilidad Ambiental y manejo de residuos en sistemas de producción de cacao en el suroriente de Boyacá-Colombia
}

\section{Environmental sustainability and waste management in cocoa production systems in the southwest of Boyacá-Colombia}

Andrés Felipe Vargas Martínez ${ }^{1}$ Javier Augusto López Cifuentes ${ }^{2}$ Álvaro Enrique Alvarado Gaona ${ }^{3}$

DOI: https://doi.org/10.19053/01228420.v18.n3.2021.12896

RESUMEN: El objetivo del presente estudio fue conocer cuál es el concepto de sostenibilidad y cómo se realiza el manejo de los residuos agrícolas (fitosanitarios, fertilizantes, biomasa e inertes) dentro de unidades productoras de cacao en el suroriente del departamento de Boyacá. Para tal fin se realizó una investigación descriptiva, no probabilística, aplicando un instrumento tipo encuesta a 52 unidades de producción de cacao de los municipios de Miraflores, Santamaría, y Campohermoso; pertenecientes a 3 asociaciones cacaoteras. Los datos revelaron que existe una concepción de la sostenibilidad ligada al concepto de crecimiento económico de corte neoliberal, en donde la sostenibilidad ambiental no es la prioridad, como sí lo es el incremento de las utilidades del negocio. Por su parte, el manejo del recurso hídrico no es adecuado, no se cuenta con agua potable y no se realiza tratamiento de las aguas residuales, las cuales no tiene ningún proceso de purificación antes de ser devueltas a los afluentes hídricos. Adicionalmente, las personas viven y laboran en el mismo sitio de trabajo. En cuanto al manejo de los residuos agrícolas, se evidencia que no existe conocimiento ni aplicación de las Buenas Prácticas Agrícolas, esto hace que se apliquen productos fitosanitarios y fertilizantes sin la supervisión técnica, sino bajo el criterio determinado por la experiencia que tenga el productor en la actividad agropecuaria.

PALABRAS CLAVES: Theobroma cacao, BPA, Producción Agrícola, Desarrollo Sostenible
ABSTRACT: The objective of this research was to know what the concept of sustainability is and how the management of agricultural residues (phytosanitary, fertilizers, biomass and inert residues) is carried out within cocoa production units in the southwest of the department of Boyacá. For this purpose, a descriptive, non-probabilistic research was carried out, applying a survey-type instrument to 52 cocoa production units in the municipalities of Miraflores, Santamaría, and Campohermoso; units belonging to 3 cocoa associations. The data revealed that there is a conception of sustainability linked to the concept of neoliberal economic growth, where environmental sustainability is not the priority, as if it is the increase in profits. On the other hand, the management of the water resource is not adequate, there is no drinking water and there is no treatment of the wastewater, which does not have any purification process before being returned to the water tributaries. Additionally, people live and work in the same workplace. Regarding the management of agricultural residues, it is evidenced that there is no knowledge or application of Good Agricultural Practices, this means that phytosanitary products and fertilizers are applied without technical supervision, the management criterion being the experience of the producer.

KEYWORDS: Theobroma cacao, GAP, Agricultural production, Sustainable development 


\section{Introducción}

El concepto de sostenibilidad ambiental tiene que ver con el desarrollo rural, territorial y humano, dado que el territorio es el espacio donde las personas construyen relaciones entre sí y con el ambiente. Por esta razón se deja de considerar el ambiente como un sujeto pasivo donde simplemente se realizan las actividades humanas, a entenderlo como un ente dinámico donde interactúan distintos tipos de seres vivos, junto con el hombre (Sepúlveda, 2016).

Igualmente, el concepto de sostenibilidad adquiere una doble connotación, dado que las actividades humanas tienen un impacto sobre los recursos existentes en el territorio, por tanto, su concepción no está separada de la economía, sino que se proyecta más ampliamente tratando de entender las actividades y características biológicas de los ecosistemas territoriales, buscando saber cómo funciona el ecosistema, para que su aprovechamiento económico necesario para la subsistencia humana tenga el menor impacto. De esta forma, tiene que ver con el principio de la escasez de los recursos, la población que existe y la presión que ejerce sobre el territorio, los procesos de contaminación y agotamiento de los recursos naturales, y las estrategias de producción limpia, en cualquiera de los campos económicos (Bravo \& Bravo, 2016; Labandeira et al., 2007; Zarta, 2018). De esta forma, Sepúlveda (2016), define la sostenibilidad como la habilidad de lograr una prosperidad económica sostenible en el tiempo, protegiendo al mismo tiempo los sistemas naturales del planeta y proveyendo una alta calidad de vida para las personas

Los mecanismos o recursos existentes para lograr un control de la contaminación proveniente de las actividades económicas pueden ser de tipo político, y corresponder al nivel público o al privado. Igualmente pueden comprender distintos tipos de instrumentos como lo son los de comando y control, donde se encuentran las normas de emisión y tecnológicas; los instrumentos de mercado o económicos como lo son los impuestos o los subsidios que desestimulan un comportamiento y estimulan otro, respectivamente. También se encuentran los instrumentos de provisión directa del estado, que se refieren a mecanismos que el gobierno provee para el manejo de residuos y desechos, así como del desarrollo tecnológico y servicios para que esto se realice (Sánchez, 2002).

El propósito de la presente investigación consistió en desarrollar una aproximación descriptiva acerca de las prácticas básicas relacionadas con el concepto de sostenibilidad ambiental, aplicadas en unidades productivas cacaoteras de municipios del suroriente de Boyacá. Para ello, se construyó un diagnóstico de sostenibilidad, indagando prácticas que tienen que ver con la sostenibilidad ambiental y económica de las unidades productoras de cacao, con la finalidad de observar, de una forma sencilla, cómo se practica en dichas comunidades el concepto de sostenibilidad ambiental. 


\section{Materiales y Métodos}

De acuerdo con Hernández y Mendoza (2018), la investigación desarrollada es de tipo descriptiva con alcance exploratorio, que emplea una metodología cualitativa e inductiva, de carácter no probabilístico; observando las características relacionadas con la sostenibilidad ambiental que poseen 52 unidades productoras de cacao ubicadas en los municipios de Campohermoso, Miraflores y Santamaría, al suroriente del departamento de Boyacá.

Se aplicó una encuesta a las unidades de estudio, en la que se evaluó las prácticas que fomentan la sostenibilidad en el sistema productivo de cacao, valorando la pertinencia del concepto, el manejo del recurso hídrico, el manejo de los residuos sólidos; la forma en que realizan la fertilización del suelo y el uso de agroquímicos. Dicho instrumento se aplicó una vez las personas firmaron un consentimiento informado, tratando los datos con reserva de identidad. La información obtenida fue analizada de forma descriptiva, mediante Microsoft Excel.

\section{Resultados y Discusión}

\section{A. Concepto de sostenibilidad}

Acerca del concepto de sostenibilidad, dentro de las unidades productivas es predominante la orientación de los productores hacia la obtención de utilidades, entendiéndolas como mayores ingresos que costos, seguido por la intención de mantenerse en el mercado en el tiempo; mediante la explotación de la tierra para la producción de cacao (Figura 1). El componente de preservación del ambiente frente al concepto de sostenibilidad es menor dentro de las unidades investigadas.

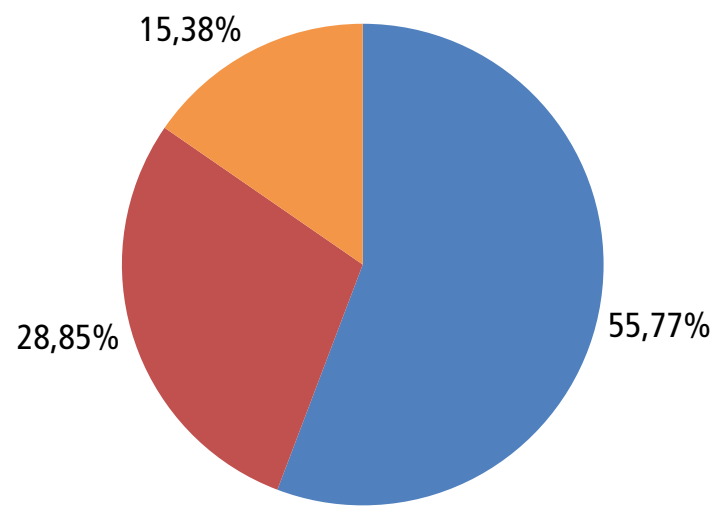

\footnotetext{
- Obtener ingresos por encima de los costos de manera que se obtenga utilidad a través del tiempo

- Perdurar en el mercado

- Satisfacer las necesidades de las generaciones presentes sin comprometer el ambiente y las posibilidades del futuro para atender sus propias necesidades en el ámbito social y económico
}

FIGURA 1. Orientación del concepto de sostenibilidad dentro de las unidades productivas cacaoteras. 


\section{B. Manejo del recurso hídrico}

Para observar los comportamientos asociados al manejo del agua, se observó las fuentes de agua usadas, encontrando que el agua lluvia es la principal fuente de abastecimiento de las comunidades, mientras que el agua potable es a la que menos acceso tienen (Figura 2).

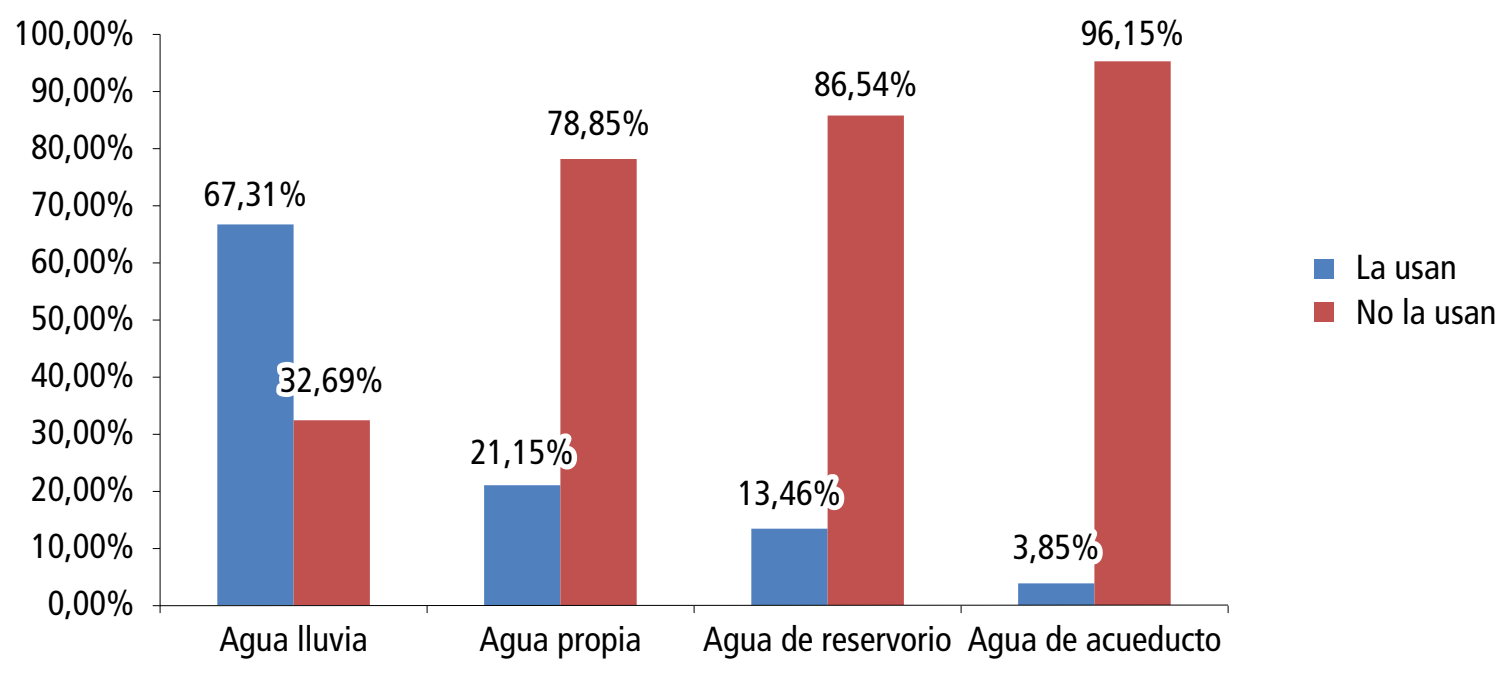

FIGURA 2. Fuentes del agua usada.

Acerca del tratamiento que le dan al agua, tanto al ingreso a los sistemas productivos como en su egreso, se observa que es muy bajo (Figura 3)

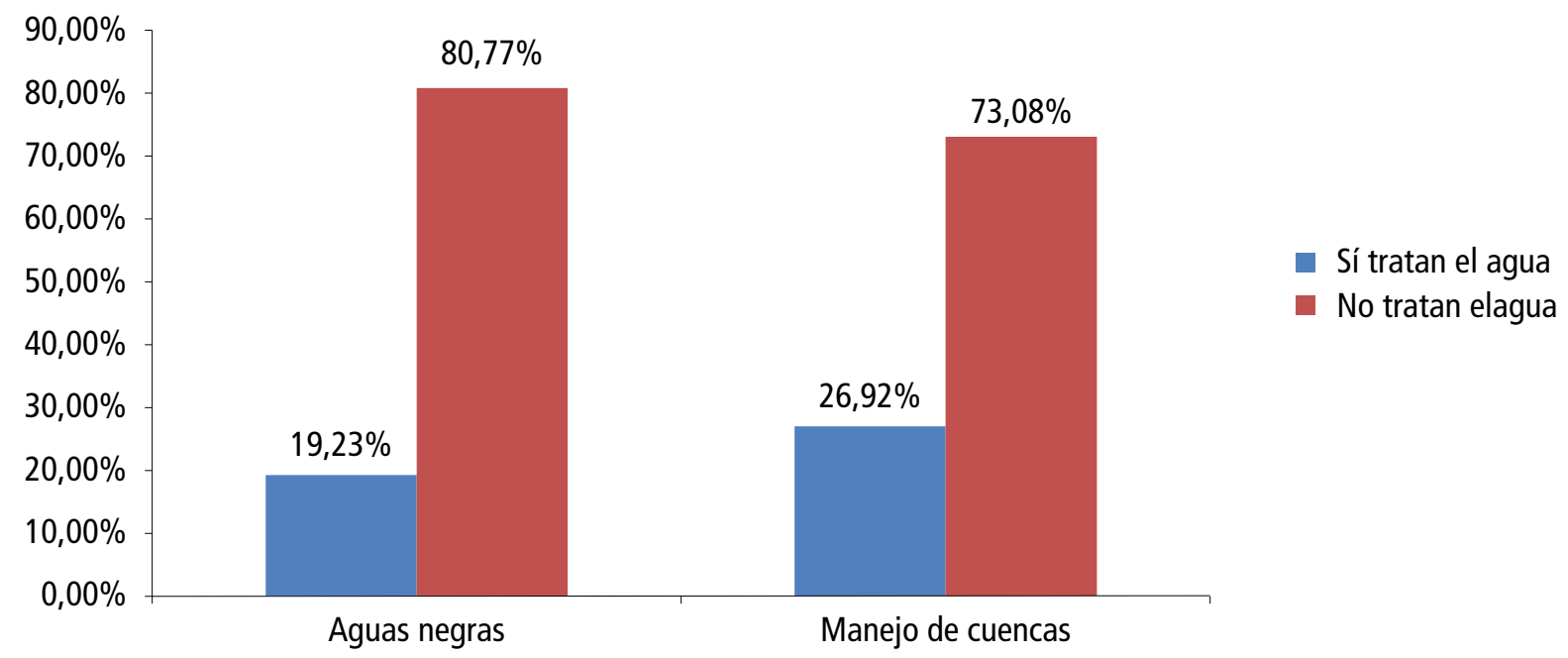

FIGURA 3. Tratamiento del agua usada.

Por otra parte, la mayor parte de las aguas residuales de los sistemas productivos son tratadas a través de pozos sépticos (Figura 4). 


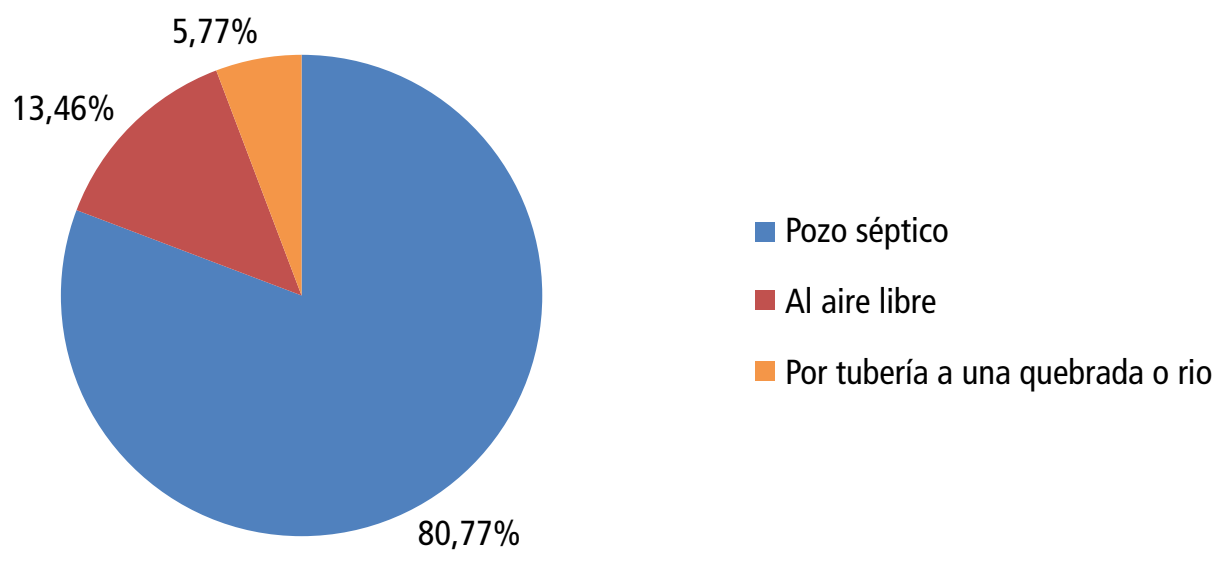

FIGURA 4. Disposición de aguas residuales.

\section{Manejo de residuos agrícolas}

Coronado y Valencia (2015); clasifica los residuos agrícolas como fitosanitarios, fertilizantes, biomasa y residuos inertes. Basándose en esa clasificación se presentan los siguientes resultados.

Acerca del uso de productos fitosanitarios, se encontró que existe una alta intensidad de uso, la cual no tiende a disminuir (Figura 5).

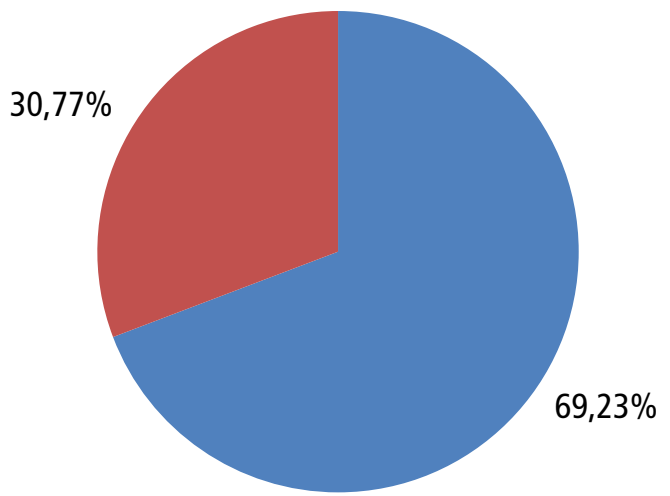

no busca minimizar el uso de productos fitosanitarios

- Busca minimizar el uso de productos fitosanitarios

FIGURA 5. Uso de agroquímicos.

Los criterios para emplear los agroquímicos obedecen principalmente a la práctica que tienen los productores, dada por los años de trabajo (Figura 6).

Adicionalmente, se halló que los tipos de agroquímicos más usados son los insecticidas, en combinación con fungicidas o solos (Figura (7).

Acerca del manejo de los fertilizantes, se investigaron los criterios que usan para abonar, los cuales corresponden a distintos niveles de combinación de variables que en su mayoría están basados en el juicio propio o criterio que da la experiencia, en forma parecida al criterio para el manejo de productos fitosanitarios (Figura 8). 


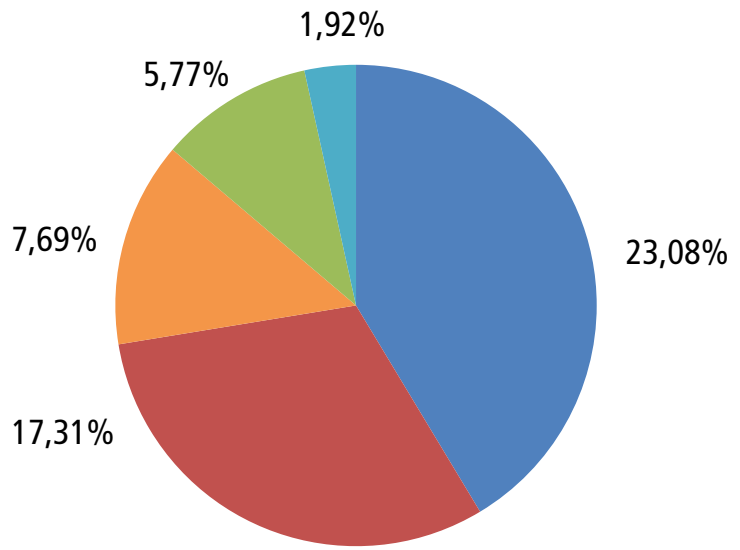
Usan productos fitosanitarios "a ojo"
- Usan productos fitosanitarios mediante evaluación agronómica
- Usan productos fitosanitarios "a ojo" y mediante evaluación de inicidencias
Usan productos fitosanitarios "a ojo" y mediante evaluación agronómica
Usan productos fitosanitarios mediante evalua- ción de inicidencias

FIGURA 6. Criterios para el empleo de agroquímicos.

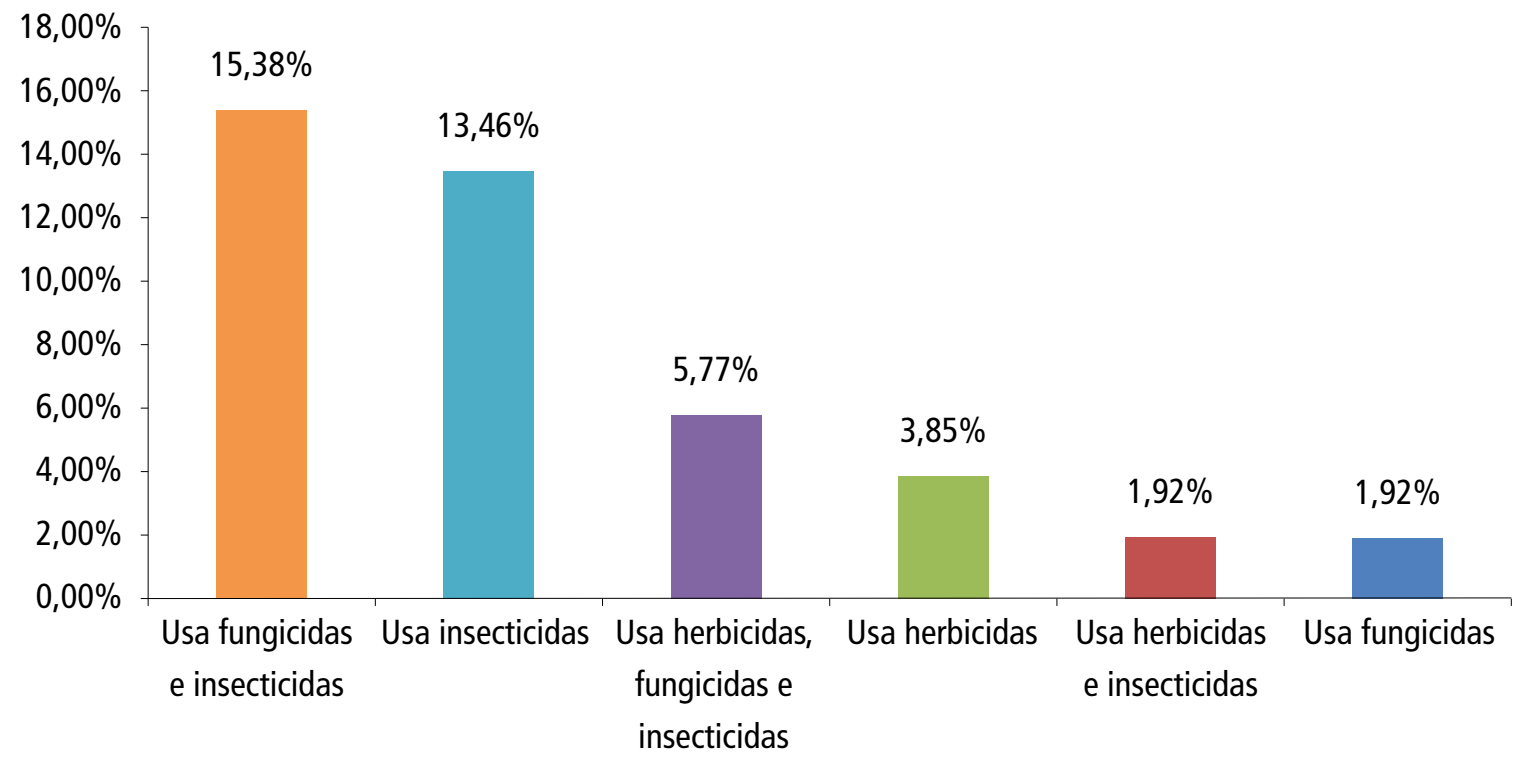

FIGURA 7. Tipos de agroquímicos usados.

Situación climática + juicio propio + análisis de suelos + Estado de desarrollo del cultivo Situación climática + juicio propio + Estado de desarrollo del cultivo Estado de desarrollo del cultivo Análisis de suelos Situación climática +juicio propio Juicio propio análisis de suelos + Estado de desarrollo del cultivo Situación climática +juicio propio + análisis de suelos juicio propio + Estado de desarrollo del cultivo juicio propio + análisis de suelos Situación climática Situación climática + análisis de suelos

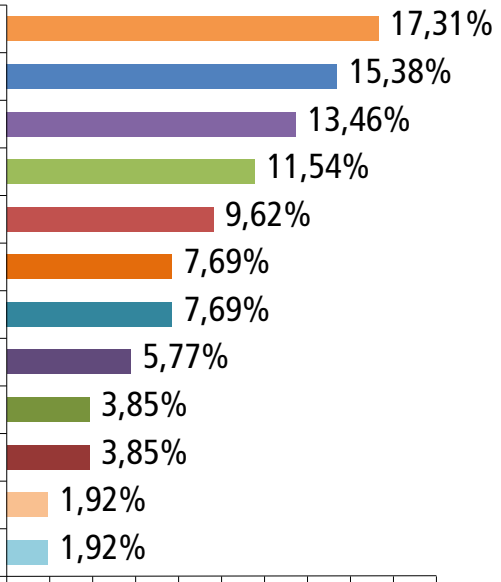

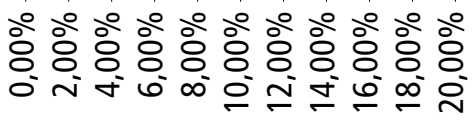

FIGURA 8. Criterios usados para abonar. 
Los tipos de abonos que usan, en su mayoría, son una mezcla de fertilizantes comerciales y de fertilizantes orgánicos, seguida por el empleo único de fertilizantes orgánicos (Figura 9)

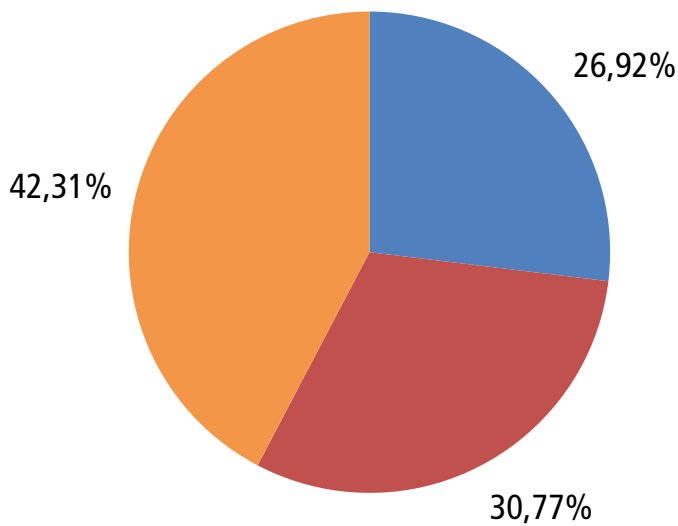

\author{
- Usa Fertilizantes químicos \\ Usa Fertilizante orgánico \\ Usa ambos tipos de fertilizantes
}

FIGURA 9. Tipos de abonos usados.

El compostaje es la mayor fuente de abono orgánico, seguida por el humus, y por último una combinación entre compostaje y humus (Figura 10)

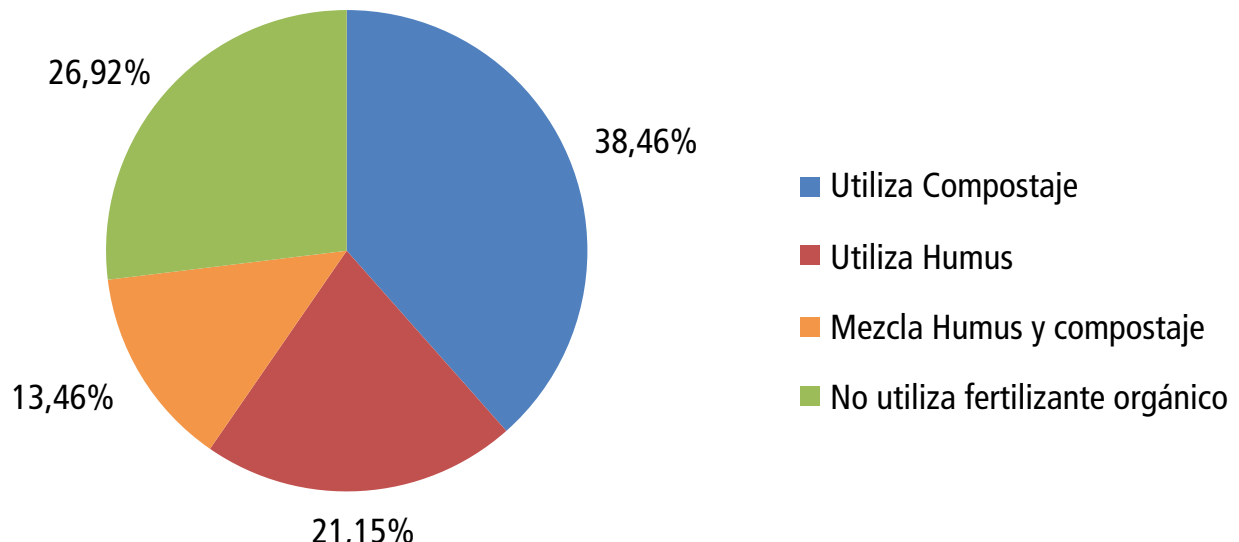

FIGURA 10. Tipo de abonos orgánicos usados.

En cuanto al manejo de biomasa y residuos inertes, los desechos del cultivo como restos de cosechas y maleza erradicada manualmente, es mayoritariamente utilizada como abonado verde para la producción de fertilizantes orgánicos y abonado de los terrenos; mientras que los recipientes plásticos son reciclados. En cambio, los residuos sólidos como excretas no tienen manejo específico, salvo el depósito en pozo séptico de las excretas humanas (Figura 11) 


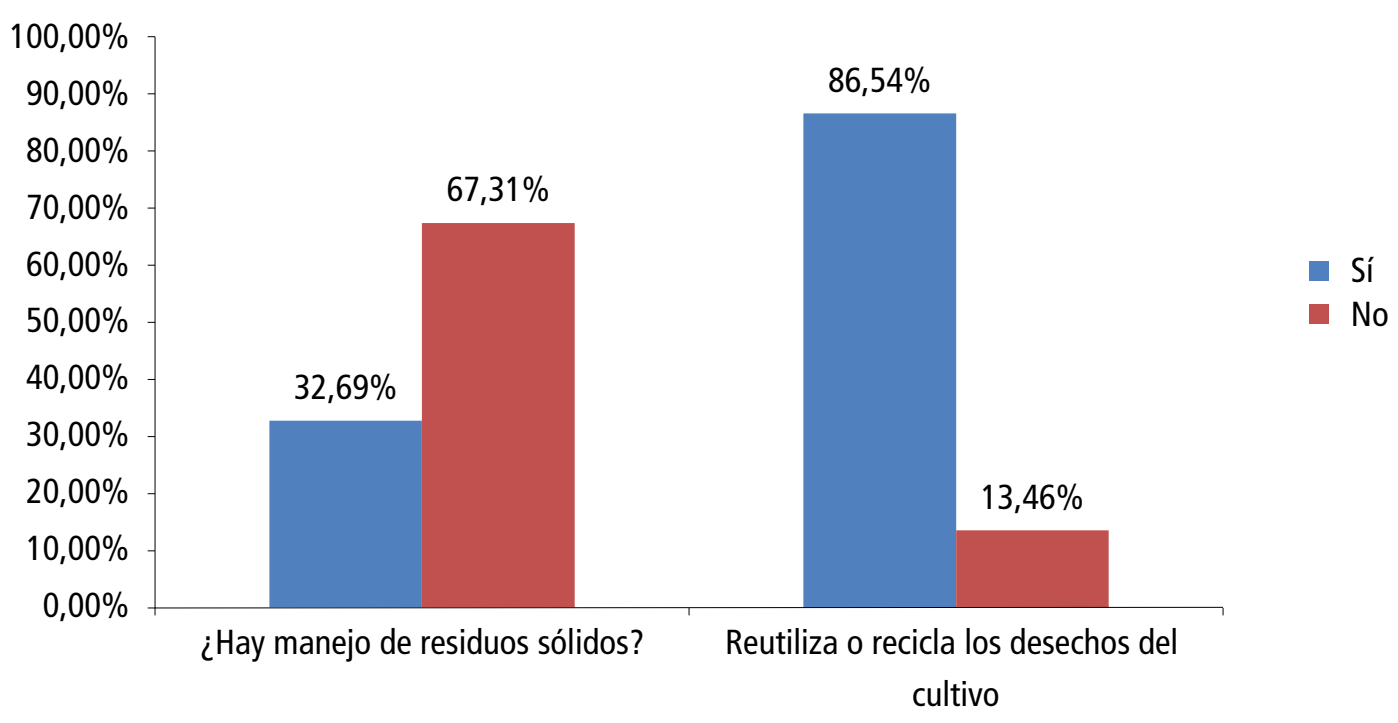

FIGURA 11. Manejo de residuos sólidos en las actividades productivas.

Por su parte, la cultura alrededor del manejo de los residuos sólidos está orientada hacia su disposición al relleno sanitario, seguida por la práctica de enterramiento y posteriormente la quema. En este ítem, a diferencia del anterior en cuanto a los desechos del cultivo, los desechos de la cotidianidad se aprovechan en menor forma, dado que se reciclan en su mayoría (Figura 12).

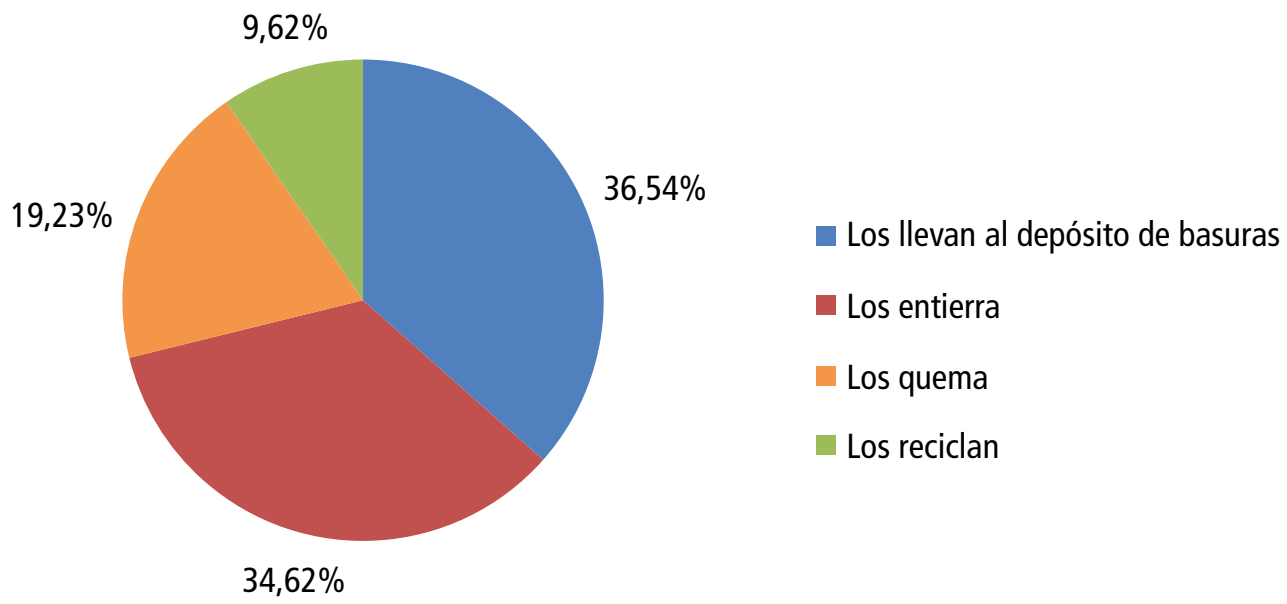

FIGURA 12. Disposición de residuos en las actividades productivas.

El pensamiento económico capitalista busca acumular y reproducir el capital, procurando maximizar los beneficios en el menor tiempo posible (SegreIles, 2008). Este modelo, intensificado desde su profundización neoliberal, ha generado una crisis global, dado que para aumentar la productividad y por ende la oferta de productos en un mundo globalizado que actúa bajo el paradigma del libre mercado, se han empleado tecnologías que potencian los ritmos de explotación de los recursos -limitados- que se encuentran en 
la naturaleza, particularmente en el mundo rural y que corresponden a la materia prima que se transforma para los demás bienes, y a los productos minero-energéticos que se transforman para propiciar la energía para los servicios. En medio de esta dinámica, se contaminan y degradan los recursos naturales y el medio, lo cual pone en peligro el equilibrio vital, al afectar el agua y la tierra, dificultando la regeneración de los ecosistemas (Segrelles, 2008).

Lo que se observa normalmente en las producciones minifundistas establecidas en Boyacá, es una economía campesina donde el autoconsumo y la subsistencia son imperantes. En estas economías se integran tanto la actividad productiva como la naturaleza, en una unidad donde se genera una conciencia ambiental y de cuidado por el entorno (Santacoloma, 2015). Sin embargo, la presión capitalista ha propiciado una transición desde esta economía hacia una donde la generación de utilidades cobra mayor importancia que la preservación del ambiente.

Frente a estas prácticas poco sostenibles, en las últimas décadas ha cobrado mayor importancia el manejo ambiental a nivel internacional y a nivel local, tanto que la construcción jurídica colombiana ha venido desarrollando importantes avances al respecto, desde los derechos y deberes consagrados constitucionalmente en la carta política de 1991, que han venido dando lugar a una "Constitución ecológica".

La Constitución política de Colombia vigente desde 1991, establece en su capítulo tercero los derechos colectivos y del ambiente (Asamblea Nacional Constituyente, 1991), lo cual, sin perjuicio del derecho al trabajo y de la libertad en cuanto a la dedicación económica, es un derrotero de obligatorio cumplimiento, dado que es en el territorio y sobre los recursos donde se desarrollan las actividades humanas, por tanto, es imprescindible hacer un uso adecuado y racional de los mismos.

Esta doctrina constitucionalmente, ha sido reafirmada con jurisprudencia como el fallo de Tutela T-411 de 1992; y las sentencias Constitucionales C-431 de 2002; C-750 de 2008; C-595 de 2010; C-123 de 2014; C-449 de 2015; C-041 de 2017 y C-219 de 2017 (Robledo \& Cifuentes, 2018), reafirmando la existencia de derechos individuales y colectivos que son objeto de garantía constitucional y legal (Vargas, 2018).

Con respecto al agua, es considerada tanto un derecho individual como un derecho colectivo, ligada a la vida y la salud del entorno, de los seres vivos y de los seres humanos. Por tal razón la Corte Constitucional de Colombia ha generado jurisprudencia donde ordena medidas para la conservación las de las fuentes hídricas con el fin de garantizar su disponibilidad en las futuras generaciones (Vargas, 2018). Su aprovisionamiento es dispuesto, de acuerdo con lo dispuesto en el artículo 365 de la Constitución Política de Colombia, 
puede ser hecho por el Estado, directa o indirectamente, por comunidades organizadas, o por particulares (Congreso de la República de Colombia, 1991). La prestación del servicio está regulada por medio de las leyes 142 y 143 de 1994.

En el caso de estudio, se observa que el origen del agua usada es principalmente de tipo agua lluvia, siendo muy valioso para los productores el agua que se almacena en su propiedad. También acceden al agua a través de reservorios, ya sean propios o de terceros. El uso del agua que proviene del acueducto es muy reducido (3,85\%), lo que implica que la mayoría del agua usada no ha sido intervenida con fines de potabilización, por tanto, el agua se usa en las explotaciones pecuarias no necesariamente se puede usar para el autoconsumo, debido a que no garantiza las condiciones organolépticas que le permita ser segura.

De otro lado, de acuerdo con Silva (2019), existen actores, prácticas y comportamientos alrededor del recurso hídrico que ejercen presiones sobre las condiciones de calidad del agua, como receptora de vertimientos dentro de las dinámicas comunitarias, incluidas las actividades agrícolas. Una de las consecuencias del mal manejo del recurso hídrico consiste en la disminución de la calidad y disponibilidad de agua, debido a la contaminación y eutrofización de fuentes hídricas por mal manejo de desechos de tipo orgánico y agropecuario, incluidos antibióticos, agroquímicos y fertilizantes (Coy, 2018).

En cuanto a la intervención para minimizar el impacto ambiental a través de planes de manejo del recurso hídrico en las unidades productivas analizadas es muy baja, siendo destinadas las aguas negras a cuencas sin darles ningún tipo de tratamiento. Igualmente, las cuencas no son intervenidas con actividades de reforestación, cuidado y/o limpieza, lo que pone en riesgo la disponibilidad del agua en condiciones óptimas para su aprovechamiento, además del desbalance ecosistémico que supone su mal manejo. Adicionalmente, para el consumo humano dentro de las unidades productivas el agua no es potable ni ha sido tratada.

Los fertilizantes, el estiércol y los plaguicidas son las principales causas de contaminación del agua (FAO, 2002). Dentro de los sistemas productivos, junto con la obtención de un producto final que se comercializa, se producen residuos derivados de la actividad agrícola iniciada para lograr dicho producto. Coronado y Valencia (2015), establecen que dichos residuos provocan una contaminación que, por la dificultad de localizar sus fuentes y su alto grado de dispersión se denomina difusa.

Los contaminantes se pueden clasificar en cuatro subtipos, los cuales son a) fitosanitarios (se refiere a los residuos de sustancias químicas utilizadas para la eliminación de seres vivos que compiten con el hombre por los recursos agrarios, tradicionalmente denominadas plagas), b) fertilizantes (debido a su 
mal uso, que muchas veces es dado por una incorrecta interpretación de las condiciones del suelo, bajo empleo de análisis de suelos, uso intensivo del material, entre otras causas, lo cual conlleva a contaminar las aguas superficiales y profundas) c) biomasa residual: provienen de seres vivos, se generan en grandes cantidades y están muy dispersos en el espacio; y su degradación es lenta, lo cual altera los ciclos en las producciones agrícolas y d) Residuos Inertes: plásticos, sustratos artificiales, embaces metálicos o plásticos, cartón, restos de maquinaria, etc (Coronado \& Valencia, 2015).

Acerca del manejo de productos fitosanitarios, en la mayoría de las unidades productivas investigadas, no se busca reducir el uso de agroquímicos. El principal criterio empleado para el empleo de agroquímicos es el criterio de la experiencia propia del productor, el cual mediante un método intuitivo decide "a ojo" aplicar el agroquímico que cree que es adecuado para una situación particular. Los principales agroquímicos usados son los insecticidas, solos o en combinación con fungicidas y herbicidas. Este empleo mixto obedece a la necesidad de aumentar la productividad para tener competitividad, premisa que se ha venido adoptando dentro de las producciones.

De acuerdo con Gil et al. (2019), los peligros biológicos para el cultivo de cacao más frecuentes en Colombia son la Hormiga arriera (Atta spp), Perforador de la mazorca (Carmenta foraseminis), Monalonion (Monalonion spp), Monilia (Moniliophthora roreri), Escoba de bruja (Moniliophthora perniciosa) y Fitóptora (Phytophthora spp). Estos se controlan con los denominados plaguicidas, que son de tipo fungicida e insecticida. Igualmente se encuentran las malezas que son controladas con herbicidas. Todos estos productos comerciales a su vez, además de las sustancias químicas vertidas en aire, suelo y agua; producen desechos de tipo plástico, por los envases en los que se venden.

Por su parte, la contaminación causada por fertilizantes se da por un uso intensivo que supera la capacidad de absorción que tiene el suelo, por el efecto de escorrentía del agua que los lixivia y los conduce a fuentes hídricas, por traslado por la acción del viento antes de ser absorbidos, o por una mezcla de los tres fenómenos. En este proceso, los fertilizantes normalmente se acumulan por acción de gravedad y se trasladan a través de fuentes hídricas a través de suelo hacia las aguas subterráneas o directamente a las fuentes perimetrales de cuerpos de agua. Esta acumulación de fertilizantes propicia la eutrofización del agua, la aparición de especies que desplazan el ecosistema natural, opalizan la luz de los cuerpos de agua y son capaces de poner en riesgo la vida de las especies endémicas, que antes permanecía en equilibrio ecosistémico (FAO, 2002).

Con respecto a la fertilización de los cultivos, dentro de las unidades productivas, se observa que existen diversas modalidades para evaluar la necesidad de abonar. El principal criterio se basa en la experiencia, es decir el juicio 
propio en combinación con la observación del Estado de desarrollo del cultivo, entre otros. Aproximadamente el $34 \%$ de las unidades productivas mezclan cuatro o tres de los cuatro criterios indagados, para realizar el proceso de fertilización.

El uso del análisis del suelo, que es un criterio objetivo, es menor. Esto puede deberse al costo y a la baja disposición de capacidad instalada para realizar laboratorios de suelo en la zona. La institucionalidad en ese sentido es precaria y la oferta privada igualmente, la cual llega solamente a través de los promotores de venta de los productos, quienes realizan charlas informativas sobre las ventajas de los productos, la forma de usarlos y las ventajas económicas y promociones existentes para adquirirlos.

Se observa que los productores asociados acuden al empleo de mezcla de abonos orgánicos y fertilizantes industriales para realizar los procesos de fertilización del suelo. Un 30\% confía en los abonos orgánicos únicamente, mientras que un $27 \%$ lo hace con fertilizantes industriales como único método.

Por su parte, el manejo de biomasa residual en cuanto a los restos de cosechas y malezas, el cual es adecuado, pues se incorpora en la producción de abonos. Las excretas en su mayoría son tratadas a través de un pozo séptico, mientras que prácticas como dejarlas expuestas al aire libre o desecharlas hacia una fuente hídrica son menos comunes. Este manejo minimiza un poco el impacto proveniente de la actividad humana. Sin embargo, es insuficiente, pues el control sobre las prácticas de fumigación y contaminación mediante el empleo de agroquímicos es prácticamente nulo, lo que podría ocasionar contaminación de las fuentes de agua, incluso subterráneas. Los residuos inertes como plásticos y son reciclados en su mayoría, lo cual es positivo.

Al observar el manejo que se hace de los residuos agrícolas en las unidades productivas se debe establecer puntualmente la incidencia que tiene en la contaminación de las aguas subterráneas del área analizada, puesto que, según, la FAO (2002), "la contaminación de las aguas subterráneas por los productos y residuos agroquímicos es uno de los problemas más importante en casi todos los países desarrollados y, cada vez más, en muchos países en desarrollo".

A pesar de la intención de los productores de realizar producciones sostenibles, el enfoque es limitado al facto económico, ante lo cual es necesario tener reconocer que el pensamiento económico capitalista de corte neoliberal ha generado una crisis global, dado que para aumentar la productividad y por ende la oferta de productos en un mundo globalizado que actúa bajo el paradigma del libre mercado, se han empleado tecnologías que potencian los ritmos de explotación de los recursos -limitados- que se encuentran en la naturaleza, particularmente en el mundo rural y que corresponden a la 
materia prima que se transforma para los demás bienes, y a los productos minero-energéticos que se transforman para propiciar la energía para los servicios. En medio de esta dinámica, se contaminan y degradan los recursos naturales y el medio, lo cual pone en peligro el equilibrio vital, al afectar el agua y la tierra, dificultando la regeneración de los ecosistemas (Segrelles, 2008 pp. 12-15).

\section{Conclusiones}

Dentro de las unidades productivas analizadas existe una transición desde la economía campesina hacia la economía capitalista, donde bajo una premisa de incremento de la riqueza monetaria se pone en riesgo el equilibrio y la sostenibilidad de los sistemas

Sumado a esta relación con el entorno natural, la sostenibilidad en términos organizacionales es precaria, lo cual pone a las unidades productivas en mayor desventaja, tanto a corto, como mediano y largo plazo, puesto que, de acuerdo con Arrarte (2005), tomar decisiones con el fin único y exclusivo de alcanzar mayores utilidades es un error, máximo cuando no se conocen los objetivos de la organización, lo cual puede incrementar el riesgo de disolución de las asociaciones en el tiempo.

Es importante incorporar dentro de las comunidades conceptos de economía ambiental, desarrollo sostenible, conservación y salud. Mientras el concepto tradicional de economía se limita al manejo óptimo de recursos limitados, la producción de bienes y servicios y su distribución (de Juan \& Mochón, 2006; Samuelson \& Nordhaus, 2010), el concepto de economía ambiental lo amplía, puesto que incorpora los elementos de sostenibilidad ambiental, de la mano del desarrollo sostenible que la ONU promueve a través de la agenda de desarrollo sostenible. Este desarrollo sostenible implica la incorporación de políticas que promuevan la salud, el desarrollo de entornos sanos y libres de contaminación, el desarrollo de tecnologías libres de emisión, la producción con menores impactos sobre la naturaleza, la reducción de la pobreza y la disminución del hambre, entre otros objetivos.

\section{Referencias}

Arrarte, R. A. (2005). ¿Maximización de utilidades o incremento del valor del accionista? Quipukamayoc, 12(24), 41-43. https://doi.org/10.15381/quipu. v12i24.5438

Bravo, M., \& Bravo, S. (2016). La economía ambiental y ecológica relacionada con el desarrollo económico. Revista DELOS: Desarrollo Local Sostenible, 25. http:// www.eumed.net/rev/delos/25/economia.html 
Congreso de la República de Colombia. (1991). Constitución Política de Colombia. http://www.secretariasenado.gov.co/senado/basedoc/constitucion_politica_1991_pr007.html\#219

Coronado, R., \& Valencia, R. A. (2015). Gestión Integral de Residuos Agrícolas para la Generación de Materias Primas en el Municipio de Cota Cundinamarca [Universidad Distrital Francisco José de Caldas]. https://repository.udistrital.edu.co/bitstream/handle/11349/3001/CoronadoGutierrezRaul2015. pdf? sequence $=1$ \&isAllowed $=y$

Coy, J. (2018). Evaluación multifuncional de explotaciones agrícolas de tipo familiar y/o campesina en el municipio de Útica, Cundinamarca [Universidad de la Salle]. https://ciencia.lasalle.edu.co/cgi/viewcontent.cgi?article=1543\&context =economia

De Juan, R., \& Mochón, A. (2006). Principios de Economía. Libro de Problemas (Tercera Edición). McGraw-Hill Interamericana de España, S.A.U.

Delegatarios a la Asamblea Nacional Constituyente. (1991). Constitución Política de Colombia del 13 de junio de 1991. [Legal]. SUIN-Juriscol. http://www.suin-juriscol.gov.co/viewDocument.asp?ruta=Constitucion/1687988

FAO. (2002). Agricultura mundial: Hacia los años 2015/2030 (Primera). FAO. http:// www.fao.org/3/y3557s/y3557s00.htm\#TopOfPage

Gil Alejandro, León Andrés, Aguirre Carlos, Suárez Darliston, Martínez Helí, Bautista Hernando, Urrego Jhorman, Puerta Jorge, Gavanzo Jorge, Valenzuela Juan, Calderón Luis, López Mauricio, Hincapié Oscar, Hernández Pablo, \& Restrepo Tatiana. (2019). Buenas prácticas agrícolas en el cultivo, beneficio y comercialización de cacao (Theobroma cacao L.) (Tercera edición). Compañía Nacional de Chocolates S.A.S. https://repository.agrosavia.co/bitstream/handle $/ 20.500 .12324 / 35708 / 35708$. pdf?sequence=1 \&isAllowed=y

Hernández, R., \& Mendoza, C. P. (2018). Metodología de la investigación: Las rutas cuantitativa, cualitativa y mixta. McGraw-Hill Interamericana de editores, S.A. de C.V.

Labandeira, X., León, C., \& Vázquez, M. X. (2007). Economía ambiental. Pearson Education.

ONU. (2015). La Asamblea General adopta la Agenda 2030 para el Desarrollo Sostenible. Desarrollo Sostenible. https://www.un.org/sustainabledevelopment/ es/20 15/09/la-asamblea-general-adopta-la-agenda-2030-para-el-desarroIlo-sostenible/

Robledo, P., \& Cifuentes, S. (2018). La jurisprudencia constitucional colombiana sobre el pago por servicios ambientales. Anuario Iberoamericano de Justicia Constitucional, 22, 263-278. https://doi.org/10.18042/cepc/aijc.22.09

Samuelson, P. A., \& Nordhaus, W. D. (2010). Economía. Con Aplicaciones a Latinoamérica (Décimonovena edición). McGraw-Hill Interamericana de editores, S.A. de C.V.

Sánchez, G. (2002). Desarrollo y medio ambiente: Una mirada a Colombia. Economía y desarrollo, 1(1), 79-98. http://uacl fuac.edu.co/revista/M/seis.pdf

Santacoloma, L. E. (2015). Importancia de la economía campesina en los contextos contemporáneos: Una mirada al caso colombiano. Entramado, 11 (2), 38-50. https://doi.org/10.18041/entramado.2015v11n2.22210 
Segrelles, J. A. (2008). La ecología y el desarrollo sostenible frente al capitalismo: Una contradicción insuperable. http://rua.ua.es/dspace/bitstream/10045/10882/1/ Microsoft\%20Word\%20-\%20Art.\%20Ecolog\%c3\%ada\%20_Brasil_.pdf

Sepúlveda, J. (2016). Sustentabilidad ambiental: Referente esencial para el desarrollo regional. Producción + Limpia, 1 1(2), 7-8. http://repository.lasallista.edu. co:8080/ojs/index.php/pl/article/view/1234/1025

Silva, C. (2019). Practicas participativas de cultura ambiental del recurso hídrico entorno al río Subachoque en Cundinamarca Colombia implementadas por la comunidad. https://ridum.umanizales.edu.co/xmlui/bitstream/handle/20.500.12746/3387/Edith\%20Catherine\%20Nin\%CC\%830\%20Silva_\%20 24_05_2018.pdf?sequence $=1$ \&isAllowed $=y$

Vargas, R. M. (2018). El derecho de la gestión comunitaria del agua en Colombia para garantizar el acceso al agua potable. Misión Jurídica, 11(15), 321-334. https://www.revistamisionjuridica.com/wp-content/uploads/2020/09/16.-ELDERECHO-DE-LA-GESTION-COMUNITARIA-DEL-AGUA-EN.pdf

Zarta, P. (2018). La sustentabilidad o sostenibilidad: Un concepto poderoso para la humanidad. Tabula Rasa: revista de humanidades, 28, 409-423. https://doi. org/10.25058/20112742.n28.18 
